\title{
PRIME IDEALS IN MATRIX RINGS
}

\author{
by ARTHUR D. SANDS \\ (Received 18th October, 1955)
}

1. Introduction. Let $R$ be a ring and let $R_{n}$ be the complete ring of $n \times n$ matrices with coefficients from $R$.

If $A$ is any subset of $R$, we denote by $A_{n}$ the subset of $R_{n}$ consisting of the matrices of $R_{n}$ with coefficients from $A$.

If $R$ is a ring with a unit element, the ideals $\dagger$ of $R_{n}$ are the sets $A_{n}$ corresponding to the ideals $A$ of $R$. But if $R$ has no unit element, this is not, in general, the case. It is however possible to establish for any ring $R$, with or without a unit element, results corresponding to the above one for two special types of ideals, namely, prime ideals and prime maximal ideals. Thus in $\S 2$ it is shown that the prime and prime maximal ideals of $R_{n}$ are the sets $A_{n}$ corre. sponding to the prime and prime maximal ideals $A$ of $R$.

In $\S 3$ it is shown that if $M$ is the $M$-radical of $R$, as defined by M. Nagata ((2), p. 338), then the $M$-radical of $R_{n}$ is $M_{n}$.

In $\S 4$ it is shown that those maximal ideals of $R_{n}$ which are of the form $A_{n}$ are the sets $A_{n}$ corresponding to the prime maximal ideals $A$ of $R$, i.e., they are the prime maximal ideals of $R_{n}$.

An ideal $P$ in a general ring $R$ is said to be prime if the following condition is satisfied; if $A$ and $B$ are ideals of $R$ such that $A B \subseteq P$, then $A \subseteq P$ or $B \subseteq P$. An ideal of $R$ is said to be semi-prime if it is an intersection of prime ideals of $R$. In (1), theorem $1, N$. H. McCoy gives a set of alternative necessary and sufficient conditions that an ideal should be prime; we shall make use of several of these conditions in our proofs. We shall also make use of the fact that any ring $R$ can be embedded in an over-ring $(1, R)$ such that $R$ is an ideal in $(1, R)$, and $(1, R)$ has a unit element. We shall also use a result of M. Nagata ((2), remark 2, p. 333) which states that if $S$ is an ideal in the ring $R$ and $A$ is a semi-prime ideal in the ring $S$, then $A$ is an ideal in $R$.

Throughout the paper we shall denote by $[r]^{i, j}$ the matrix which has $r$ as its $(i, j)$ th coefficient and has all its other coefficients equal to zero.

2. Prime and prime maximal ideals in $R$.

Theorem 1. The prime ideals of $R_{n}$ are the sets $A_{n}$ corresponding to the prime ideals $A$ of $R$.

Proof: We first show that if $A$ is a prime ideal of $R$, then $A_{n}$ is a prime ideal of $R_{n}$. If $A$ is any ideal of $R$, it is easily seen that $A_{n}$ is an ideal of $R_{n}$. Let $A$ be a prime ideal of $R$. Let $\left[a_{i j}\right]$ and $\left[b_{i j}\right]$ be matrices of $R_{n}$ such that $\left[a_{i j}\right] R_{n}\left[b_{i j}\right] \subseteq A_{n}$. Suppose that $\left[a_{i j}\right] \notin A_{n}$. Let $a_{k l}$ be a coefficient of $\left[a_{i j}\right]$ which is not contained in $A$. Let $r$ be any element of $R$ and $b_{p q}$ any coefficient from $\left[b_{i j}\right]$. Then $\left[a_{i j}\right][r]^{l, p}\left[b_{i j}\right]$ is a matrix of $\left[a_{i j}\right] R_{n}\left[b_{i j}\right]$ which has the element

$\dagger$ Throughout this paper, ideal will mean two.sided ideal.

† For the sake of completeness we give the proof of this result.

Since a semi-prime ideal is an intersection of prime ideals, it is sufficient to prove the corresponding result for prime ideals.

Let $S$ be an ideal in a ring $R$ and $A$ a prime ideal in the ring $S$. The ideal in $R$ generated by $A$ is $A+R A+A R+R A R$. Since $S$ is an ideal of $R$ containing $A, S$ contains $A+R A+A R+R A R$. This ideal of $R$ is a fortiori an ideal of $S$; hence $S(A+R A+A R+R A R) S \subseteq S A S \subseteq A$. But $A$ is a prime ideal in $S$. 'Therefore $A+R A+A R+R A R \subseteq A$. It follows that $A$ is an ideal in $R$.

This completes the proof. 
$a_{k l} r b_{p q}$ as its $(k, q)$ th coefficient. But $\left[a_{i j}\right] R_{n}\left[b_{i j}\right] \subseteq A_{n}$; therefore $a_{k l} r b_{p q} \epsilon A$. This is true for each element $r$ in $R$; hence $a_{k l} R b_{p q} \subseteq A$. But $A$ is a prime ideal and $a_{l k l} A$; it follows by con. dition (3) of (1), theorem 1 , that $b_{p q} \in A$. This is true for each coefficient of $\left[b_{i j}\right]$; hence $\left[b_{i j}\right] \in A_{n}$. Thus, if $\left[a_{i j}\right] R_{n}\left[b_{i j}\right] \subseteq A_{n}$ and $\left[a_{i j}\right] \notin A_{n}$, it follows that $\left[b_{i j}\right] \in A_{n}$. Therefore $A_{n}$ is a prime ideal in $R_{n}$.

We now show that every prime ideal of $R_{n}$ is of this form. Let $A^{*}$ be a prime ideal in $R_{n}$. We denote by $A$ the set of elements of $R$ which are coefficients in matrices of $A^{*}$.

$R$ is an ideal in the ring $(1, R)$. Therefore $R_{n}$ is an ideal in $(1, R)_{n} . A^{*}$ is a prime ideal in $R_{n}$. Hence, by the result of Nagata, $A^{*}$ is an ideal in $(1, R)_{n}$. But $(1, R)$ has a unit element. It follows that $A$ is an ideal in $(1, R)$ and that $A^{*}=A_{n}$. But the elements of $A$ are contained in $\mathrm{R}$; hence $A$ is an ideal in $R$.

It remains to show that $A$ is prime in $R$. Let $a$ and $b$ be elements of $R$ such that $a R b \subseteq A$. Then $[a]^{1,1} R_{n}[b]^{1,1} \subseteq A_{n}=A^{*}$. But $A^{*}$ is prime in $R_{n}$; therefore $[a]^{1,1}$ or $[b]^{1,1}$ is contained in $A_{n}$. Hence $a$ or $b$ is contained in $A$. It follows that $A$ is a prime ideal in $R$.

This completes the proof.

Since an ideal $A$ in $R$ is different from an ideal $B$ in $R$ if and only if $A_{n}$ is different from $B_{n}$, it follows that the mapping $A \rightarrow A_{n}$ sets up a one-to-one correspondence between the prime ideals of $R$ and of $R_{n}$.

CoROLLARX. The semi-prime ideals of $R_{n}$ are the sets $A_{n}$ corresponding to the semi-prime ideals $A$ of $R$.

TheOREM 2. The prime maximal ideals of $R_{n}$ are the sets $A_{n}$ corresponding to the prime maximal ideals $A$ of $R$.

Proof: We first show that the prime maximal ideals of $R_{n}$ are of this form. Let $A^{*}$ be a prime maximal ideal of $R_{n}$ and let $A$ be defined as in theorem 1. Then, since $A^{*}$ is prime, it follows from theorem 1 that $A$ is a prime ideal in $R$ and that $A^{*}=A_{n}$. Let $B$ be an ideal of $R$ which strictly contains $A$. Then $B_{n}$ strictly contains $A_{n}$. Hence, by the maximality of $A_{n}$, $B_{n}=R_{n}$. Therefore $B=R$. It follows that $A$ is a maximal ideal of $R$.

It remains to show that every ideal of this form in $R_{n}$ is a prime maximal ideal. Let $A$ be a prime maximal ideal of $R$. Then, by theorem $1, A_{n}$ is a prime ideal of $R_{n}$. Let $B^{*}$ be an ideal of $R_{n}$ which strictly contains $A_{n}$. Then the set $B$ strictly contains the ideal $A$. Let $b$ be an element of $B$ which is not an element of $A$. Then, since $A$ is prime, $R b R$ is not contained in $A$; for $R b R \subseteq A$ implies that $b R b R \subseteq A$. By condition (4) of (1), theorem 1, it follows that $b R \subseteq A$ and hence that $b R b \subseteq A$. But from this it follows that $b \in A$. Thus $R b R$ is not contained in $A$. But $R b R$ is an ideal and $A$ is a maximal ideal. Hence $A+R b R=R$. Thus if $r$ is any element of $R$, there exist an element $a$ of $A$ and elements $s_{k}$ and $r_{k}$ of $R$ such that $r=a+\Sigma_{k} b r_{k}$. Let $\left[r_{i j}\right]$ be any matrix of $R_{n}$. Then for each pair of integers $i, j$ there exist an element $a_{i j}$ of $A$ and elements $s_{i j k}$ and $r_{i j k}$ of $R$ such that $r_{i j}=a_{i j}+\sum_{i} s_{i j k} b r_{i j k}$. Thus

$$
\left[r_{i j}\right]=\left[a_{i j}+\sum_{k} s_{i j k} b r_{i j k}\right]=\left[a_{i j}\right]+\sum_{i, j}\left[\underset{k}{\sum s_{i j k}} b r_{i j k}\right]^{i, j} .
$$

Let $b^{*}$ be a matrix of $B^{*}$ with $b$ as a coefficient, say in the $(p, q)$ th position. Then $\left[r_{i j}\right]=\left[a_{i j}\right]+\sum_{i, j k} \sum_{k}\left[s_{i j k}\right]^{i, p} b^{*}\left[r_{i j k}\right]^{q, j}$. But $B^{*}$ is an ideal of $R_{n}$; therefore $\left[s_{i j k}\right]^{i, p} b^{*}\left[r_{i j k}\right]^{0, j}$ is an element of $B^{*}$. Now $\left[a_{i j}\right]$ is an element of $A_{n}$ and so of $B^{*}$. It follows that $\left[r_{i j}\right]$ is an element of $B^{*}$. But this is true for each matrix $\left[r_{i j}\right]$ of $R_{n}$. Therefore $B^{*}=R_{n}$. Hence $A_{n}$ is a maximal ideal and so a prime maximal ideal of $R_{n}$. 
This completes the proof.

3. The M-radical of a matrix ring $R_{n}$.

M. Nagata defined the $M$-radical of a ring $R$ to be the intersection of all prime ideals $A$ of $R$ such that $R / A$ is a simple ring. It is easily seen that this is just the intersection of all prime maximal ideals of $R . \dagger$ We now use theorem 2 to show that the usual relationship between the radical of a ring $R$ and the radical of $R_{n}$ holds for the $M$-radical. $M_{n}$.

Theorem 3. If $M$ is the $M$-radical of a ring $R$, then the $M$-radical $M\left(R_{n}\right)$ of $R_{n}$ is equal to

Proof: Let $A_{\alpha}$ be the complete set of prime maximal ideals of $R$. Then, by theorem $2,\left(A_{a}\right)_{n}$ is the complete set of prime maximal ideals of $R_{n}$. Hence

$$
M\left(R_{n}\right)=\cap\left(A_{\alpha}\right)_{n}=\left(\frown A_{\alpha}\right)_{n}=M_{n} .
$$

4. Maximal ideals in $R_{n}$.

Lemma. Let $A$ be a maximal ideal in a ring $R$. Then $A$ is a prime ideal if and only if $R^{2}$ is not contained in $A$.

Proof: Let $A$ be prime. Then $R^{2} \subseteq A$ implies that $R \subseteq A$. This is not so, since $A$ is maximal. Hence $R^{2}$ is not contained in $A$.

Conversely, suppose that $R^{2}$ is not contained in $A$. Let $B$ and $C$ be ideals such that $B C \subseteq A$. If neither $B$ nor $C$ is contained in $A$, it follows from the maximality of $A$ that $B+A=C+A=R$. In this case $R^{2}=(B+A)(C+A) \subseteq B C+A \subseteq A$. But this contradicts the hypothesis that $R^{2}$ is not contained in $A$. Hence either $B$ or $C$ is contained in $A$. Therefore $A$ is prime.

Theorem 4. Let $A$ be a maximal ideal in a ring $R$. Then $A_{n}$ is a maximal ideal in $R_{n}$ if and only if $A$ is a prime ideal in $R$.

Proof: Let $A$ be a prime ideal in $R$; then, by theorem $2, A_{n}$ is a prime maximal ideal in $R_{n}$.

Conversely, suppose that $A$ is not a prime ideal. Then, by the lemma, $R^{2} \subseteq A$. Therefore $R_{n}{ }^{2} \subseteq A_{n}$. Let $b$ be an element of $R$ which is not an element of $A$. Consider the set $B^{*}$ of $R_{n}$. consisting of all matrices of the form $\left[a_{i j}\right]+[k b]^{1,1}$, where $\left[a_{i j}\right]$ is a matrix of $A_{n}$ and $k$ is an integer. Then $B^{*}$ is strictly contained in $R_{n}$ and strictly contains $A_{n}$. Clearly the difference of two matrices of $B^{*}$ is a matrix of $B^{*}$. Also

$$
R_{n} B^{*} \subseteq R_{n}^{2} \subseteq A_{n} \subseteq B^{*} \text { and } B^{*} R_{n} \subseteq R_{n}^{2} \subseteq A_{n} \subseteq B^{*}
$$

Therefore $B^{*}$ is an ideal in $R_{n}$. Hence $A_{n}$ is not a maximal ideal in $R_{n}$.

This completes the proof.

\section{REFERENCES}

(1) N. H. MoCoy, Prime Ideals in General Rings, Amer. J. Math., 71, 1949, 823-833.

(2) M. Nagata, On the Theory of Radicals In a Ring, J. Math. Soc. Jap., 3, 1951, 330-344.

\section{The UnIVersity,} Glasaow

+ We adopt the convention that the intersection of an empty set of ideals is the whole ring $R$. 